\title{
Echocardiographic Findings and Genotypes in Autosomal Dominant Polycystic Kidney Disease
}

\author{
Ryohei Miyamoto $^{a} \quad$ Akinari Sekine $^{a, b}$ Takuya Fujimaru ${ }^{c}$ Tatsuya Suwabe ${ }^{a, b}$

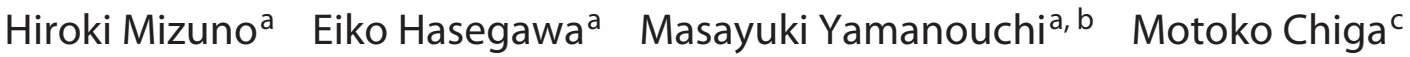

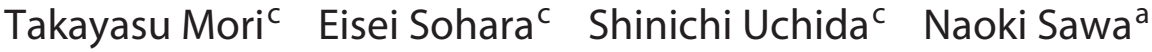 \\ Yoshifumi Ubara $^{a, b}$ Junichi Hoshino ${ }^{a, b}$ \\ ${ }^{a}$ Nephrology Center, Toranomon Hospital, Tokyo, Japan; ${ }^{b}$ Okinaka Memorial Institute for Medical Research, \\ Toranomon Hospital, Tokyo, Japan; 'Department of Nephrology, Graduate School of Medical and Dental Sciences, \\ Tokyo Medical and Dental University, Tokyo, Japan
}

\section{Keywords}

Autosomal dominant polycystic kidney disease P PKD1 . PKD2 - Mitral regurgitation · Cardiovascular disease

\begin{abstract}
Background: Autosomal dominant polycystic kidney disease (ADPKD) is the most common hereditary cystic kidney disease and is well known to have extrarenal complications. Cardiovascular complications are of particular clinical relevance because of their morbidity and mortality; however, unclear is why they occur so frequently in patients with ADPKD and whether they are related to the genotypes. Methods: We extracted and retrospectively analyzed clinical data on patients with ADPKD who underwent echocardiography and whose genotype was confirmed by genetic testing between April 2016 and December 2020. We used next-generation sequencing to compare cardiac function, structural data, and the presence of cardiac valvular disease in patients with 1 of 3 genotypes: PKD1, PKD2, and non-PKD1, 2. Results: This retrospective study included 65 patients with ADPKD. Patients were divided into 3 groups: $P K D 1, n=32 ; P K D 2, n=$
\end{abstract}

karger@karger.com www.karger.com/kdd

Karger" BOPEN ACCESS
(C) 2021 The Author(s)

Published by S. Karger AG, Basel

This is an Open Access article licensed under the Creative Common Attribution-NonCommercial-4.0 International License (CC BY-NC) (http://www.karger.com/Services/OpenAccessLicense), applicable to the online version of the article only. Usage and distribution for commercial purposes requires written permission.
12; and non-PKD1, 2, $n=21$. The prevalence of mitral regurgitation (MR) was significantly higher in the PKD1 group than in the PKD2 and non-PKD1, 2 group ( $46.9 \%$ vs. $8.3 \%$ vs. $19.0 \%$, respectively; $p=0.02$ ). In contrast, no significant difference was found for other cardiac valve complications. Conclusion: This study found a significantly higher prevalence of MR in patients with the PKD1 genotype than in those with the PKD2 or non-PKD1, 2 genotypes. Physicians may need to perform echocardiography earlier and more frequently in patients with ADPKD and the PKD1 genotype and to control fluid volume and blood pressure more strictly in these patients to prevent future cardiac events.

(C) 2021 The Author(s).

Published by S. Karger AG, Base

\section{Introduction}

Autosomal dominant polycystic kidney disease (ADPKD) is the most common hereditary cystic kidney disease, affecting 1 in 1,000-2,500 people worldwide [1]. ADPKD has been linked to mutations in 2 genes: PKD1 gene (chromosome 16; 16p13.3) and PKD2 gene (chro-

Correspondence to:

Akinari Sekine, akinari-s@ toranomon.gr.jp 


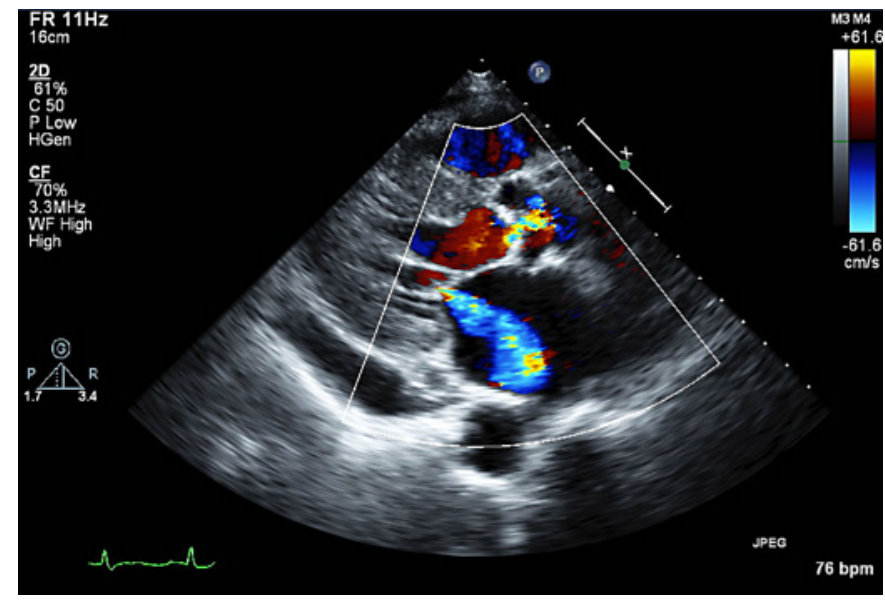

Fig. 1. Typical picture of mitral regurgitation.

mosome 4; 4q21); the former mutation was found in $78 \%$ of patients and the latter in 15\% [1]. Approximately, 7\% of patients with ADPKD appear not to have a PKD1/2 mutation.

ADPKD is also well known for its extrarenal complications, including cardiovascular diseases (CVDs), such as intracranial aneurysm, hypertension, cardiac valve-related disease, left ventricular hypertrophy (which is related to changes in some other parameters, such as an increase of the left ventricular mass index [LVMi]), arrhythmia, and cardiomyopathy [2-7]. These CVDs are considered to be the most common cause of death in patients with ADPKD, so evaluation and intervention are clinically important. Noteworthy is that mitral valve-related complications such as mitral valve prolapse (MVP) and mitral regurgitation (MR) have been reported to occur frequently in patients with ADPKD $[8,9]$. MR in particular can lead to arrhythmia, left heart dilation, cardiac system dysfunction, and heart failure, so clinicians should evaluate patients and intervene as necessary to improve patient prognosis. MR is typically observed from the parasternal long-axis view of transthoracic echocardiography, as shown in Figure 1.

The reason why mitral valve-related complications occur more frequently in patients with ADPKD is still unclear. Previous studies found that patients with ADPKD tend to develop hypertension more frequently and earlier than healthy individuals $[5,10,11]$. Also, patients with ADPKD may be more prone to fluid overload than healthy individuals because they experience progressive renal dysfunction at a younger age [2]. The guidelines from the American College of Cardiology/American
Heart Association (ACC/AHA), European Society of Cardiology/European Association for Cardio-Thoracic Surgery (ESC/EACTS), and Japanese Circulation Society (JCS) dictate that blood pressure and fluid volume management are important to prevent the occurrence and worsening of MR because the early onset of hypertension and renal dysfunction in ADPKD is hypothesized to account for the high prevalence of MR; however, a causal relationship has not been shown. Moreover, Lumiaho et al. [12] reported that patients with ADPKD with the PKD1 mutation had higher rates of MVP and MR than healthy individuals, but the specific characteristics that are associated with the high rate of MR in patients with ADPKD remain unclear.

In this study, we aimed to identify the relationship between cardiac valvular diseases and genetic mutations in ADPKD by extracting and retrospectively analyzing clinical data on patients with a confirmed genetic profile who underwent echocardiography. To our knowledge, no other study has compared the incidence of cardiac valvular events between genetic mutations in ADPKD. Thus, the study should answer the question whether all or only a subgroup of patients with ADPKD should routinely undergo echocardiography and whether such a subgroup can be identified by specific characteristics such as blood pressure, previous history of CVDs, or genotype.

\section{Materials and Methods}

\section{Patients}

This study included 68 patients with ADPKD who underwent echocardiography and genetic testing between April 2016 and December 2020 at Toranomon Hospital, Tokyo, Japan. Genetic analyses were performed at Tokyo Medical and Dental University. Eligibility criteria included a diagnosis of ADPKD according to the modified Pei-Ravine criteria from the protocol of the Replicating Evidence of Preserved Renal Function: an Investigation of Tolvaptan Safety and Efficacy in ADPKD trial. Genotype could not be confirmed in 3 patients, so we excluded them from the study.

\section{Renal Function}

The Evidence-based Clinical Practice Guideline for Chronic Kidney Disease from the Japanese Society of Nephrology diagnoses chronic kidney disease (CKD) on the basis of estimated glomerular filtration rate (eGFR) and proteinuria. The eGFR was calculated by the Japanese eGFR equation [13]. We used eGFR as an objective criterion of renal function and to categorize patients into the following groups: G1, eGFR >90; G2, eGFR from 60 to 89; G3a, eGFR from 45 to 59; G3b, eGFR from 30 to 44; G4, eGFR from 15 to 29; and G5, eGFR <15. Category G5D was defined as patients who required hemodialysis and in whom eGFR could therefore not be calculated. 
Table 1. Clinical data and laboratory characteristics of patients with ADPKD

\begin{tabular}{lllll}
\hline & $\begin{array}{l}\text { PKD1 group } \\
(n=32)\end{array}$ & $\begin{array}{l}\text { PKD2 group } \\
(n=12)\end{array}$ & $\begin{array}{l}\text { Non-PKD1, 2 group } \\
(n=21)\end{array}$ & $p$ value \\
\hline Age, years & 59 & 59 & 63 & 0.98 \\
Male sex, $n$ (\%) & $11(34.4)$ & $5(41.7)$ & $11(52.4)$ & 0.43 \\
Family history, $n$ (\%) & $10(31.3)$ & $4(33.3)$ & $5(23.8)$ & 0.82 \\
BMl, ${ }^{* 1}$ kg/m ${ }^{2}$ & 21 & 20 & 23 & 0.01 \\
Renal function, ${ }^{2} n(\%)$ & & & \\
G1 & $2(6.5)$ & $1(8.3)$ & 0 & 0.58 \\
G2 & $5(16.1)$ & $4(33.3)$ & $6(31.6)$ & 0.34 \\
G3 & $6(19.4)$ & $1(8.3)$ & $6(31.6)$ & 0.35 \\
G4 & $4(12.9)$ & $3(25.0)$ & $2(10.5)$ & 0.56 \\
G5 & $6(19.4)$ & $1(8.3)$ & $2(10.5)$ & 0.71 \\
G5D & $8(25.8)$ & $2(16.7)$ & $3(15.8)$ & 0.71 \\
Serum creatinine, $\mathrm{mg} / \mathrm{dL}$ & 1.29 & 0.91 & 0.99 & 0.40 \\
Hypertension, ${ }^{3} n(\%)$ & $22(71.0)$ & $7(63.6)$ & $17(89.5)$ & 0.25 \\
Taking diuretics & $7(22.6)$ & $1(9.1)$ & $5(26.3)$ & 0.42 \\
Systolic blood pressure, $\mathrm{mm} \mathrm{Hg}$ & 128 & 120 & 133 & 0.15 \\
Diastolic blood pressure, $\mathrm{mm} \mathrm{Hg}$ & 80 & 77 & 83 & 0.65 \\
mBP, ${ }^{* 4}$ mm Hg & 97.0 & 90.3 & 99.3 & 0.38 \\
Total kidney volume, ${ }^{* 5} \mathrm{~mL}$ & 2,103 & 1,262 & 1,395 & 0.26 \\
CVD, $n$ (\%) & $1(3.1)$ & 0 & 0 & 1.00 \\
Diabetes, $n$ (\%) & $3(9.4)$ & $2(16.7)$ & $2(9.5)$ & 0.76 \\
Alcohol use, ${ }^{* 6} n$ (\%) & $15(50.0)$ & $5(41.7)$ & $11(55.0)$ & 0.76 \\
\hline
\end{tabular}

ADPKD, autosomal dominant polycystic kidney disease; CVD, cardiovascular disease; BMI, body mass index; $\mathrm{mBP}$, mean blood pressure; eGFR, estimated glomerular filtration rate. * ${ }^{1}$ Two patients in the PKD1 group, 1 patient in the PKD2 group, and 2 patients in the non-PKD1, 2 group lacked data on BMI. ${ }^{2}$ One patient in the PKD1 group and 2 patients in the non-PKD1, 2 group lacked data on renal function. G1, eGFR >90; G2, eGFR from 60 to 89; G3a, eGFR from 45 to 59; G3b, eGFR from 30 to 44; G4, eGFR from 15 to 29; and G5, eGFR <15. * 3 One patient in each of the $P K D 1$ and the PKD2 groups and 2 patients in the non-PKD1, 2 group lacked data on hypertension. ${ }^{*} \mathrm{mBP}$ was calculated with the following formula: $\mathrm{mBP}=$ diastolic blood pressure + (systolic blood pressure - diastolic blood pressure)/3. * ${ }^{5}$ Five patients in the PKD1 group, 2 patients in the PKD2 group, and 3 patients in the non-PKD1, 2 group lacked data on total kidney volume. ${ }^{6}$ Two patients in the PKD1 group and 1 patient in the non-PKD1,2 group lacked data on alcohol use.

\section{Hypertension}

Hypertension was defined as an office blood pressure $>140 / 90$ $\mathrm{mm} \mathrm{Hg}$ at echocardiography. This definition was based on the Japanese Society of Hypertension (JSH) Guidelines for the Management of Hypertension (JSH 2019) [14]. Patients who were prescribed any of the antihypertensive agents recommended in the guideline, such as diuretics, calcium channel blockers, renin-angiotensin system inhibitors (including angiotensin-converting enzyme inhibitors and angiotensin receptor blockers), and betablockers, were classified as having hypertension, even if the office blood pressure was below 140/90 $\mathrm{mm} \mathrm{Hg}$.

\section{Total Kidney Volume}

Total kidney volume was estimated from computed tomography scans by volume measurement software (VINCENT).

\section{Echocardiography}

All echocardiography measurements were transthoracic and were performed by certified medical technologists and cardiologists at Toranomon Hospital. If echocardiography was performed several times, the earliest data were used. The diagnosis of cardiac valvular disorders such as MR, mitral stenosis (MS), aortic regurgitation (AR), aortic stenosis (AS), tricuspid regurgitation (TR), and pulmonary regurgitation (PR) was assessed according to the guideline from the JCS at the time the echocardiography performed.

The Toranomon Hospital defines normal left ventricular enddiastolic diameter (LVDd) ranges as $34-54 \mathrm{~mm}$. Left ventricular diastolic volume (LVDv) and LVMi were calculated from the LVDd, intraventricular septum thickness, posterior left ventricular wall thickness, and body surface area. LVDd, LVDv, and LVMi were evaluated to compare cardiac structure. The left ventricular ejection fraction (LVEF), which indicates the contraction function of the heart, is estimated in the dimension M-mode or by the modified Simpson's method. If both values were available, the latter was used in the analyses. The mitral E/e' was used as a measure of the dilation function of the heart. The $\mathrm{E}$ wave is the peak mitral inflow velocity during early diastole, measured by the wave Doppler technique, and the e' velocity is the mitral annular velocity during early diastole, measured by the tissue Doppler technique [15]. 
Table 2. Echocardiographic findings in patients with ADPKD

\begin{tabular}{lllll}
\hline & $\begin{array}{l}\text { PKD1 group } \\
(n=32)\end{array}$ & $\begin{array}{l}\text { PKD2 group } \\
(n=12)\end{array}$ & $\begin{array}{l}\text { Non-PKD1, 2 group } \\
(n=21)\end{array}$ & $p$ value \\
\hline LVDd, mm & 47.0 & 43.9 & 48.7 & 0.05 \\
LVDV, $\mathrm{mL}$ & 102 & 88 & 110 & 0.11 \\
$\mathrm{LVMi},{ }^{* 7} \mathrm{~g} / \mathrm{m}^{2}$ & 91 & 70 & 73 & 0.31 \\
$\mathrm{LVEF}, \%$ & 72.2 & 69.4 & 70.0 & 0.76 \\
Mitral E/e' & 10.3 & 8.8 & 9.3 & 0.54 \\
MR, $n(\%)$ & $15(46.9)$ & $1(8.3)$ & $4(19.0)$ & 0.02 \\
$\mathrm{MS}, n(\%)$ & $2(6.3)$ & $1(8.3)$ & $1(4.8)$ & 1.00 \\
$\mathrm{AR}, n(\%)$ & $7(21.9)$ & $1(8.3)$ & $3(14.3)$ & 0.68 \\
$\mathrm{AS}, n(\%)$ & $1(3.1)$ & $0(0.0)$ & $1(4.8)$ & 1.00 \\
$\mathrm{TR}, n(\%)$ & $12(37.5)$ & $2(16.7)$ & $6(28.6)$ & 0.43 \\
$\mathrm{PR}, n(\%)$ & $15(46.9)$ & $7(58.3)$ & $6(28.6)$ & 0.22 \\
\hline
\end{tabular}

* 7 Two patients in the PKD1 group, 1 patient in the PKD2 group, and 2 patients in the non-PKD1, 2 group lacked data on LVMi due to lack of data on BMI. ADPKD, autosomal dominant polycystic kidney disease; MR, mitral regurgitation; MS, mitral stenosis; $A R$, aortic regurgitation; AS, aortic stenosis; TR, tricuspid regurgitation; PR, pulmonary regurgitation; LVDd, left ventricular end-diastolic diameter; LVDv, left ventricular diastolic volume; LVMi, left ventricular mass index; LVEF, left ventricular ejection fraction; BMI, body mass index.

\section{Genetic Analysis}

Genetic analysis was performed at Tokyo Medical and Dental University with capture-based next-generation sequencing according to the method reported previously [16-18]. The next-generation sequencing panel provided good coverage of the exonic regions of both $P K D 1$ and $P K D 2$, except for exon 1 of $P K D 1$. This approach detected no mutations of 6 pseudogenes.

\section{Statistical Analysis}

We analyzed the data of the 68 patients with ADPKD and tabulated the clinical characteristics according to the type of mutation. Categorical variables were summarized as the number (percentage) and continuous variables, as the mean with the standard deviation or median with the interquartile range (IQR), as appropriate. Patients' characteristics and laboratory findings were compared between genotypes ( $P K D 1, P K D 2$, and non-PKD1, 2) with the Kruskal-Wallis method for categorical variables and with the $\chi^{2}$ test and Fisher's exact test for continuous variables. The relationship between CKD groups and MR prevalence was evaluated with the trend test. For all analyses, $p<0.05$ was taken to indicate statistical significance. All analyses were conducted with STATA ${ }^{\circledR}$ SE version 14.0 (StataCorp, College Station, TX, USA). This study was performed in accordance with the Declaration of Helsinki and was approved by the research Ethics Committees of Toranomon Hospital and Tokyo Medical and Dental University Hospital. Written informed consent was obtained from all patients.

\section{Results}

\section{Genetic, Clinical, and Laboratory Characteristics}

The study included 65 patients, who were classified into the following 3 groups on the basis of their genotypes:
PKD1 group $(n=32)$, PKD2 group $(n=12)$, and non$P K D 1,2$ group $(n=21)$. The characteristics of these groups at the time of echocardiography are shown in Table 1. All participants were Japanese, and we found no significant differences between the groups regarding age, sex, family history, renal function (number of patients in categories G1-G5, patients who required hemodialysis, and serum creatinine), hypertension, the number of patients who were prescribed diuretics, blood pressure (systolic pressure, diastolic pressure, and mean blood pressure (mBP), CVD, diabetes, or alcohol use ( $p>0.05)$; however, the non$P K D 1,2$ group had a higher body mass index (BMI) than the other 2 groups (each $p=0.01$ ). No patient had ischemic heart disease or cardiomyopathy, but 1 patient in the PKD1 group had undergone aortic arch replacement for aortic dissection before the first echocardiography.

The following data were missing in some patients: BMI, 5 patients (which meant that LVMi could not be calculated in these patients); renal function, 3 patients; hypertension, 4 patients; TKV, 10 patients; and alcohol use, 3 patients. Detailed information on the genetic variants detected in the patients is presented in online supplementary Tables 1 and 2 (for all online suppl. material, see www.karger.com/doi/10.1159/000520300).

\section{Influence of Genotype on Cardiac Valvular Diseases in $A D P K D$}

The echocardiographic findings of all groups are shown in Table 2. MR was documented in 15 patients of 


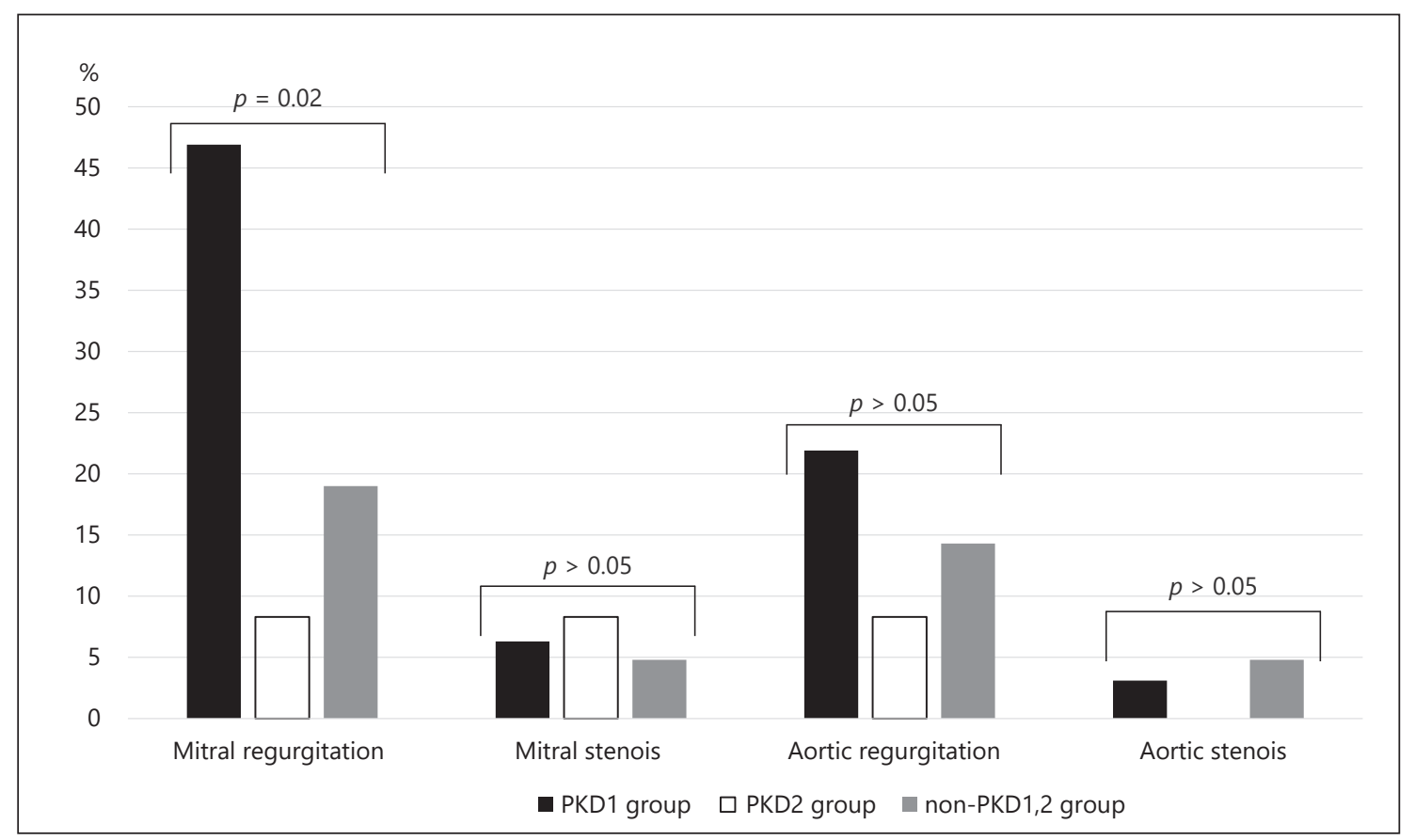

Fig. 2. Prevalence of cardiac valvular complications.

the $P K D 1$ group, 1 patient of the $P K D 2$ group, and 4 patients of the non-PKD1, 2 group. The prevalence of MR was significantly higher in the $P K D 1$ group than in the $P K D 2$ and non- $P K D 1,2$ groups ( $46.9 \%$ vs. $8.3 \%$ vs. $19.0 \%$, respectively; $p=0.02$ ) (Table 2; Fig. 2). No patient in any of the 3 groups appeared to have MVP. MS was found in 2 patients in the $P K D 1$ group and 1 patient each in the $P K D 2$ and non-PKD1, 2 groups; AR, in 7 patients in the $P K D 1$ group, 1 patient in the $P K D 2$ group, and 3 patients of the non-PKD1, 2 group; AS, in 1 patient each in the $P K D 1$ and non-PKD1, 2 groups but no patient in the $P K D 2$ group; TR, in 12 patients in the $P K D 1$ group, 2 patients in the $P K D 2$ group, and 6 patients in the non- $P K D 1$, 2 group; and $\mathrm{PR}$, in all 15 patients in the $P K D 1$ group, 7 patients in the PKD2 group, and 6 patients in the non$P K D 1,2$ group. We found no significant difference between the $P K D 1, P K D 2$, and non-PKD1, 2 groups in the prevalence of MS (6.3\% vs. $8.3 \%$ vs. $4.8 \%$, respectively; $p$ $=1.00), \operatorname{AR}(21.9 \%$ vs. $8.3 \%$ vs. $14.3 \%$, respectively; $p=$ $0.68)$, AS ( $3.1 \%$ vs. $0 \%$ vs. $4.8 \%$, respectively; $p=1.00)$, TR ( $37.5 \%$ vs. $16.7 \%$ vs. $28.6 \%$, respectively; $p=0.43$ ), or PR $(46.9 \%$ vs. $58.3 \%$ vs. $28.6 \%$, respectively; $p=0.22)$ (Table 2; Fig. 2).

The median LVDd was $47.0 \mathrm{~mm}$ (IQR, 44.1-53.3 mm) in the PKD1 group, $43.9 \mathrm{~mm}$ (IQR, $40.5-46.3 \mathrm{~mm}$ ) in the
PKD2 group, and $48.7 \mathrm{~mm}$ (IQR, 45.3-52.2 $\mathrm{mm}$ ) in the non- $P K D 1,2$ group; the median LVDv was $102 \mathrm{~mL}$ (IQR, $78-135 \mathrm{~mL}$ ) in the $P K D 1$ group, $88 \mathrm{~mL}$ (IQR, $72-100 \mathrm{~mL}$ ) in the PKD2 group, and $110 \mathrm{~mL}$ (IQR, $85-127 \mathrm{~mL}$ ) in the non- $P K D 1,2$ group; the median LVMi was $91 \mathrm{~g} / \mathrm{m}^{2}$ (IQR, $67-112 \mathrm{~g} / \mathrm{m}^{2}$ ) in the PKD1 group, $70 \mathrm{~g} / \mathrm{m}^{2}$ (IQR, 56-92 g/ $\mathrm{m}^{2}$ ) in the PKD2 group, and $73 \mathrm{~g} / \mathrm{m}^{2}$ (IQR, $60-98 \mathrm{~g} / \mathrm{m}^{2}$ ) in the non-PKD1, 2 group; the median LVEF was $72.2 \%$ (IQR, 70.5-74.5\%) in the PKD1 group, 69.4\% (IQR, 67.8$75.7 \%$ ) in the PKD2 group, and 70.0\% (IQR, 66.2-75.0\%) in the non- $P K D 1,2$ group; and the median mitral E/e' was 10.3 (IQR, 8.4-12.9) in the PKD1 group, 8.8 (IQR, 8.210.1 ) in the PKD2 group, and 9.3 (IQR, 7.7-12.0) in the non- $P K D 1,2$ group. No significant differences were found in LVDd, LVDv, LVMi, LVEF, or mitral E/e' between the 3 groups.

\section{Discussion}

This cross-sectional study aimed to identify whether cardiac valvular diseases are associated with genetic mutations in ADPKD. The study found a significantly higher incidence of MR in the PKD1 group than in the $P K D 2$ and non-PKD1, 2 groups but no significant dif- 
ferences between the groups in measures of cardiac contraction and dilation function, in left ventricular dilation, or in any other cardiac valvular complications. No patient was found to have ischemic heart disease or cardiomyopathy. Furthermore, we found no significant differences between the groups in patient characteristics such as renal function and the number of patients on hemodialysis or with hypertension. There appeared to be a trend toward a higher number of patients with CKD G5 and G5D in the PKD1 group; this higher number was considered to have an impact on the increased prevalence of $\mathrm{MR}$, given that the progression of CKD is likely to cause fluid overload. We additionally examined the prevalence of MR in each CKD stage, regardless of the mutation, but found no significant difference; the prevalence of MR was $0 \%$ in CKD G1, 26.7\% in CKD G2, 38.5\% in CKD G3, 33.3\% in CKD G4, 22.2\% in CKD G5 (which does not require hemodialysis), and $38.5 \%$ in G5D (trend test, $p=0.47$ ). Therefore, our results indicate that the prevalence of MR is related to the PKD1 mutation itself.

An animal model study showed that $P k d 1$ is expressed throughout the heart, in particular at the aortic outflow, atrial appendages, endocardial cushions, and valve leaflets [19]. In contrast, another animal model study found that $P k d 2$ was highly expressed at the endocardial cushions and valve leaflets; however, the study found almost no differences in cardiac valvular insufficiencies between healthy mouse models and $P k d 2$ knockout mouse models [20]. These animal model studies may corroborate our finding of a higher prevalence of MR in the PKD1 group than in the PKD2 group.

The current study indicated that early and more frequent echocardiographic follow-up and stricter volume and blood pressure control may be justified in patients with ADPKD with the PKD1 mutation. Investigating and intervening in cardiac valve disease earlier and in a more targeted way may prevent future cardiovascular events in these patients.

The current study has some limitations. First, it was a retrospective, single-center study with a small sample size. Second, the study was cross-sectional, and echocardiographic findings should preferably be evaluated over time. Furthermore, the study may have a selection bias because we included only patients who underwent genetic testing. Genetic testing is not commonly performed in Japan, but it is often performed in patients without an apparent family history. Despite these limitations, our finding that MR occurs at a significantly higher rate in patients with the PKD1 genotype than those with the PKD2 and non-PKD1, 2 genotypes is of interest and should be further examined in a large, prospective, multicenter study.

In conclusion, we showed that the prevalence of MR is significantly higher in patients with ADPKD and the PKD1 genotype than in the other genotype groups. Physicians may need to perform echocardiography earlier and more frequently in patients with ADPKD and the PKD1 genotype and should control patients' fluid volume and blood pressure more strictly to prevent future cardiac events.

\section{Acknowledgments}

The manuscript was edited by a native English-speaking medical editor from Yamada Translation Bureau, Inc. (Tokyo, Japan).

\section{Statement of Ethics}

This study was performed in accordance with the Declaration of Helsinki and was approved by the research Ethics Committees of Toranomon Hospital and Tokyo Medical and Dental University Hospital (Approval Number 2016-9). Written informed consent was obtained from all patients.

\section{Conflict of Interest Statement}

J.H. has received a research Grant from Otsuka Pharmaceutical Co. All other authors have no competing financial interests to declare.

\section{Funding Sources}

This work was supported by a Grant-in-Aid for Scientific Research (JSPS KAKENHI Grant No. 18K08227), a research Grant from Otsuka Pharmaceutical Co., a research Grant from the Okinaka Memorial Institute to J.H., and a research Grant from the Okinaka Memorial Institute to Y.U. It was also supported in part by other Grants-in-Aid for Scientific Research (25221306, 16H05314, 19H01049, and 19H03672); Challenging Exploratory Research Grants (15K15327, 16K15467, and 18K19534); the Research Activity Start-up (17H06657, 20K22926); a Young Scientist Grant (19K17733) from the Japanese Society for the Promotion of Science; a Health Labor Science Research Grant from the Ministry of Health Labor and Welfare, AMED (Grant No. JP18ek0109304); and Grants from the Yukiko Ishibashi Foundation and the Salt Science Research Foundation $(1925,2030)$. None of the funding bodies had any role in the study design, data analysis, decision to publish, or preparation of the manuscript. 


\section{Author Contributions}

R.M., A.S., and J.H. designed the study; R.M., A.S., T.S., H.M., M.K., E.H., M.Y., N.H., N.S., and Y.U. managed the patients and collected the clinical data; R.M., A.S., and J.H. analyzed the genetic and clinical data; T.F., T.M., E.S., and S.U. analyzed the gene mutations; R.M. and A.S. prepared the figures and tables; all the authors approved the final version of the manuscript.

\section{Data Availability Statement}

All data generated or analyzed during this study are included in this article and its online supplementary Files. Further enquiries can be directed to the corresponding author.

\section{References}

1 Cornec-Le Gall E, Alam A, Perrone RD. Autosomal dominant polycystic kidney disease. Lancet. 2019;393:919-35.

2 Kuo IY, Chapman AB. Polycystins, ADPKD, and cardiovascular disease. Kidney Int Rep. 2020;5:396-406.

3 Alam A, Perrone RD. Left ventricular hypertrophy in ADPKD: changing demographics. Curr Hypertens Rev. 2013;9:27-31.

4 Helal I, Reed B, Mettler P, Mc Fann K, Tkachenko O, Yan XD, et al. Prevalence of cardiovascular events in patients with autosomal dominant polycystic kidney disease. Am J Nephrol. 2012;36:362-70.

5 Ecder T, Schrier RW. Cardiovascular abnormalities in autosomal-dominant polycystic kidney disease. Nat Rev Nephrol. 2009;5:2218.

6 Ecder T. Cardiovascular complications in autosomal dominant polycystic kidney disease. Curr Hypertens Rev. 2013;9:2-11.

7 Chebib FT, Hogan MC, El-Zoghby ZM, Irazabal MV, Senum SR, Heyer CM, et al. Autosomal dominant polycystic kidney patients may be predisposed to various cardiomyopathies. Kidney Int Rep. 2017;2:913-23.

8 Hossack KF, Leddy CL, Johnson AM, Schrier RW, Gabow PA. Echocardiographic findings in autosomal dominant polycystic kidney disease. N Engl J Med. 1988;319:907-12.
9 Timio M, Monarca C, Pede S, Gentili S, Verdura C, Lolli S. The spectrum of cardiovascular abnormalities in autosomal dominant polycystic kidney disease: a 10-year follow-up in a five-generation kindred. Clin Nephrol. 1992;37:245-51.

10 Rahbari-Oskoui F, Williams O, Chapman A. Mechanisms and management of hypertension in autosomal dominant polycystic kidney disease. Nephrol Dial Transplant. 2014; 29:2194-201.

11 Chapman AB, Stepniakowski K, RahbariOskoui F. Hypertension in autosomal dominant polycystic kidney disease. Adv Chronic Kidney Dis. 2010;17:153-63.

12 Lumiaho A, Ikäheimo R, Miettinen R, Niemitukia L, Laitinen T, Rantala A, et al. Mitral valve prolapse and mitral regurgitation are common in patients with polycystic kidney disease type 1. Am J Kidney Dis. 2001;38: 1208-16.

13 Matsuo S, Imai E, Horio M, Yasuda Y, Tomita K, Nitta K, et al. Revised equations for estimated GFR from serum creatinine in Japan. Am J Kidney Dis. 2009;53:982-92.

14 Umemura S, Arima H, Arima S, Asayama K, Dohi Y, Hirooka Y, et al. The Japanese Society of Hypertension guidelines for the management of hypertension (JSH 2019). Hypertens Res. 2019;42:1235-481.
15 Park JH, Marwick TH. Use and limitations of E/e' to assess left ventricular filling pressure by echocardiography. J Cardiovasc Ultrasound. 2011;19:169-73.

16 Sekine A, Fujimaru T, Hoshino J, Suwabe T, Oguro M, Mizuno H, et al. Genotype-clinical correlations in polycystic kidney disease with no apparent family history. Am J Nephrol. 2019;49:233-40.

17 Fujimaru T, Mori T, Sekine A, Mandai S, Chiga M, Kikuchi $\mathrm{H}$, et al. Kidney enlargement and multiple liver cyst formation implicate mutations in PKD1/2 in adult sporadic polycystic kidney disease. Clin Genet. 2018;94: 125-31.

18 Mori T, Hosomichi K, Chiga M, Mandai S, Nakaoka H, Sohara E, et al. Comprehensive genetic testing approach for major inherited kidney diseases, using next-generation sequencing with a custom panel. Clin Exp Nephrol. 2017;21:63-75.

19 Boulter C, Mulroy S, Webb S, Fleming S, Brindle K, Sandford R. Cardiovascular, skeletal, and renal defects in mice with a targeted disruption of the Pkd1 gene. Proc Natl Acad Sci U S A. 2001;98:12174-9.

20 Stypmann J, Engelen MA, Orwat S, Bilbilis K, Rothenburger M, Eckardt L, et al. Cardiovascular characterization of $\mathrm{Pkd} 2(+/ \mathrm{LacZ})$ mice, an animal model for the autosomal dominant polycystic kidney disease type 2 (ADPKD2). Int J Cardiol. 2007;120:158-66. 